\title{
URN MODELS AND BETA-SPLINES
}

\author{
R. N. Goldman \\ Control Data Corporation \\ Minneapolis, Minnesota
}

\section{Introduction}

A well-established connection exists between discrete urn models and the standard curves and surfaces used in computer-aided geometric design (CAGD) [1], [2], [3],[4],[5]. The Bézier and B-spline blending functions both model elementary stochastic processes, and many of the geometric properties of Bézier and B-spline curves and surfaces can be derived by studying these probabilistic models [4], [5]. Recently Barsky has introduced a new type of spline into CAGD called the beta-spline [6],[7],[8]. The purpose of this paper is to try to gain some insight into the properties of beta-splines by applying the techniques of urn models.

\section{Beta-Splines}

Beta-splines are generalizations of B-splines. They were developed in order to replace the somewhat artificial concept of parametric continuity by the more natural notion of geometric continuity. Briefly the idea is this: Two curves $L(t)$ $t_{0} \leq t \leq t_{1}, R(u) u_{0} \leq u \leq u_{1}$ are said to meet with $n^{t h}$-order parametric continuity $\left(C^{n}\right)$ if and only if

$$
\frac{\mathrm{d}^{\mathrm{k}}}{\mathrm{du}} \mathrm{u}^{\mathrm{k}} \mathrm{u}_{\mathrm{u}}=\frac{\mathrm{d}_{0} \mathrm{k}-\frac{\mathrm{L}}{\mathrm{k}}}{\mathrm{dt}} \mid \mathrm{t}=\mathrm{t}_{1}
$$

$$
\mathrm{k}=0,1, \ldots, \mathrm{n}
$$

Unfortunately this definition depends on more than just the geometry of the curves $L(t), R(u)$; it also depends on the specific choice of their parametric representations. A linear change of parameter $u=\beta v \beta>0$ will not change the shape of the curve $R(u)$, but by the chain rule

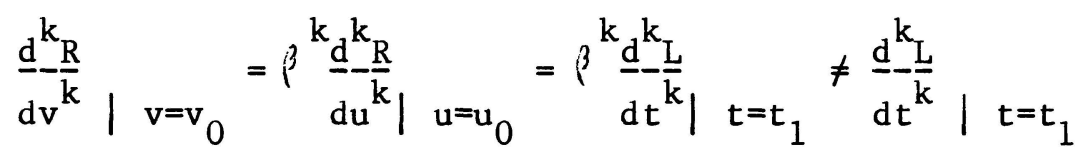

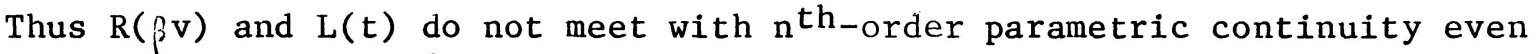
though the curves $R(\hat{\gamma}), R(u)$ are geometrically identical. To rectify this anomaly, the concept of geometric continuity is introduced.

Two curves $L(t), R(u)$ are said to meet with linear $n^{\text {th-order geometric }}$ continuity $\left(L G^{n}\right)$ if and only if there exists a constant $\beta>0$ such that

$$
\left.\frac{\mathrm{d}^{\mathrm{k}} \mathrm{R}}{\mathrm{du}}\right|_{\mathrm{u}=\mathrm{u}_{0}}=\beta^{\mathrm{k}} \stackrel{\mathrm{d}}{\mathrm{k}}-\frac{\mathrm{L}}{\mathrm{k}} \mathrm{t}^{\mathrm{t}=\mathrm{t}_{1}}
$$$$
\mathrm{k}=0,1, \ldots, \mathrm{n}
$$ 
It is easy to check that the notion of linear $n^{\text {th }}$-order geometric continuity is invariant under linear changes of parameter. Of course, this concept is not invariant under non-linear changes of parameter. A more general notion of geometric continuity $\left(G^{n}\right)$ and more general constraint equations invariant under non-linear changes of parameter are given in [8].

Splines have typically been defined in terms of parametric continuity, and the B-splines form a convenient basis for these parametric splines. The more general notion of geometric continuity requires us to search for a new set of basis functions suitable for these new types of splines. These basis functions are called beta-splines. We shall now use urn models to construct beta-splines and study their properties.

\section{An Urn Model for Beta-Splines}

Consider an urn initially containing white balls and b black balls. One ball at a time is drawn at random from the urn, its color inspected, and then returned to the urn. If the ball was the $j^{\text {th }}$ white ball to be chosen, then $\beta j(w+b)$ additional black balls are added to the urn; if the ball was the $j^{\text {th }}$ black ball to be chosen, then $\beta^{-j}(w+b)$ additional white balls are added to the urn.

We now introduce the following notation:

$$
\begin{aligned}
& t=\frac{w}{w+b}=\text { probability of selecting a white ball on the first trial } \\
& \sigma_{j}(\beta)=1+\beta+\ldots+\beta^{j-1} \\
& s_{j}^{N}(t)=s_{j}^{N}(\beta, t)=\begin{array}{l}
\text { probability of selecting a white ball after selecting } \\
\text { exactly } j \text { white balls in the first } N \text { trials }
\end{array} \\
& f_{j}^{N}(t)=f_{j}^{N}(\beta, t)=\begin{array}{l}
\text { probability of selecting a black ball after selecting } \\
\text { exactly } j \text { white balls in the first } N \text { trials }
\end{array} \\
& B_{j}^{N}(t)=B_{j}^{N}(\beta, t)=\begin{array}{l}
\text { probability of selecting exactly } j \text { white balls in the } \\
\text { first } N \text { trials }
\end{array}
\end{aligned}
$$

For each fixed $\beta$ it can be shown that the functions $B_{0}^{N}(t), \ldots, B_{N}^{N}(t)$ are linearly independent polynomials of degree $N$ and they satisfy the constraint equations $(*)$

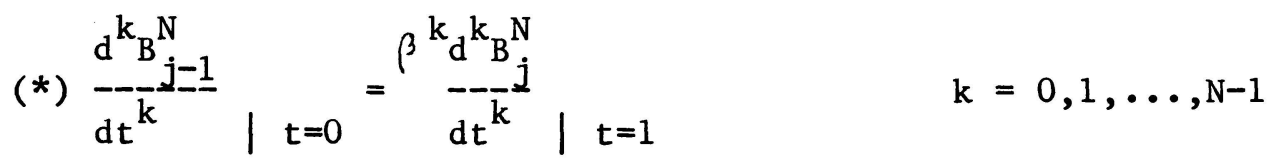

The functions $B f(t), \ldots, B N(t)$ are the beta-spline basis functions. If $\beta=1$, these functions are the uniform $B-s p l i n e$ basis functions and the urn model is the standard urn model for B-splines [1], [4] •

Given a sequence of control points $P=\left(P_{0}, \ldots, P_{M}\right)$, we can use these beta-spline basis functions as blending functions to construct $\mathrm{LG}^{\mathrm{N}-1}$ continuous beta-spline curves in much the same way that we use the uniform $B-s p l i n e$ basis functions to define $\mathrm{C}^{\mathrm{N}-1}$ continuous $\mathrm{B}-\mathrm{spline}$ curves. Define the $i^{\text {th }}$ curve 
segment by setting

$$
B_{i}[\beta, P](t)=\sum_{j=0}^{N} B_{j}^{N}(\beta, t-i) P_{i+j} \quad i \leq t \leq i+1
$$

and define the beta-spline curve by setting

$$
B[\beta, P](t)=B_{i}[\beta, P](t) \quad i \leq t \leq i+1 \quad 0 \leq i \leq M-N
$$

From the constraint equations (*) it follows immediately that

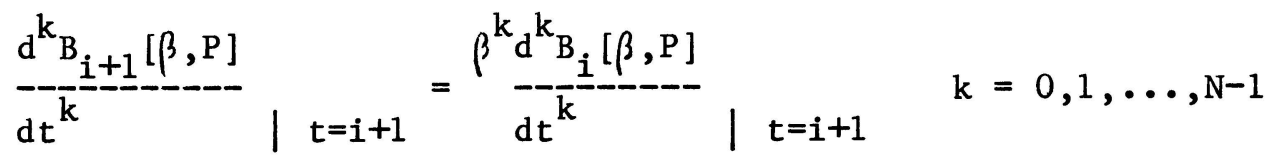

Thus $B[\beta, P](t)$ is an $L G^{N-1}$ continuous beta-spline curve.

Without moving the control points, we can alter the shape of the beta-spline curve $B[\beta, P](t)$ simply by changing the scalar parameter $\beta$. The effect of increasing $\beta$ is to move the curve closer to its control polygon and to bias the curve towards its initial control points. Thus our $\beta$ corresponds to Barsky's bias parameter $\beta_{1}[7]$.

In table 1 we summarize those properties of beta-spline basis functions and beta-spline curves which are directly derivable from the beta-spline urn model. Many of these properties are new and are presented here for the first time.

\section{Conclusion}

Urn models can be used to construct beta-spline basis functions and to derive the basic properties of these blending functions and the corresponding beta-spline curves. This is only the beginning; much work remains to be done. Here we have dealt only with the simple notion of linear geometric continuity and with the most elementary beta parameter. Non-linear geometric continuity leads to additional beta parameters and to more complicated basis functions [8]. Whether urn models can give us any insight into these higher order concepts still remains to be investigated. 


\section{References}

1. Goldman, R. N., An Urnful of Blending Functions, IEEE Computer Graphics and Applications, vo1. 3, no. 7, 1983, pp. 49-54.

2. Goldman, R. N., An Intuitive Approach to Bézier and Other Random Curves and Surfaces, SIGGRAPH Tutorial on Freeform Curves and Surfaces, 1983.

3. Goldman, R. N., Geometry and Probability, SIGGRAPH Tutorial on Freeform Curves and Surfaces, 1984.

4. Goldman, R. N., Polya's Urn Model and Computer Aided Geometric Design, SIAM Journ. on Algebraic and Discrete Methods, vol. 6, no. 1, 1985, pp. 1-28.

5. Goldman, R. N., Urn Models, Approximations, and Splines, Submitted to the Journal of Approximation Theory.

6. Barsky, B. A., The Beta-Spline: A Local Representation Based on Shape Parameters and Fundamental Geometric Measures, Ph.D. Thesis, University of Utah, Salt Lake City, Utah, 1981.

7. Barsky, B. A. and Beatty, J. C., Local Control of Bias and Tension in Beta-Splines, ACM Trans. on Graphics, vol. 2, no. 2, 1982, pp. 109-134.

8. Barsky, B. A. and DeRose, T. D., Geometric Continuity of Parametric Curves, Tech. Rept. No. UCB/CSD 84/205, Computer Science Division University of California, Berkeley, October 1984. 
TABLE 1 - PROPERTIES OF BETA-SPLINE BASIS FUNCTIONS AND CURVES

$$
\begin{aligned}
& \text { Urn Basis Functions } \quad \underline{\text { Curve }}
\end{aligned}
$$

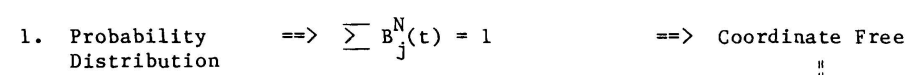

$$
\begin{aligned}
& \text { Distribution } \\
& \Rightarrow B_{j}^{N}(t) \geq 0 \quad 0 \leq t \leq 1 \quad \Rightarrow \text { Local Convex Hull Property } \\
& \text { 2. Symmetry between } \Rightarrow \text { Symmetry Formula } \quad \Rightarrow \text { Curve Symmetry } \\
& \text { white, } \beta \text { and } \quad B_{j}^{N}(\beta, t)=B_{N-j}^{N}\left(\beta^{-1}, 1-t\right) \quad B[\beta, P](t)=B\left[\beta^{-1}, P_{\text {Reverse }}\right](M-N+1-t) \\
& \text { 3. Counting } \quad \Rightarrow \text { Explicit Formulas } \quad \Rightarrow>\text { Recursion Formula } \\
& \Rightarrow \text { Locality of End Points } \\
& f_{j}^{N}(t)=\frac{\beta^{N-j}\left(\sigma_{j+1}(\beta)-t\right)}{\sigma_{N+1}(\beta)} \\
& s_{j}^{N}(t)=\frac{\beta^{N-j}+\sigma_{N-j}(\beta)}{\sigma_{N+1}(\beta)}
\end{aligned}
$$

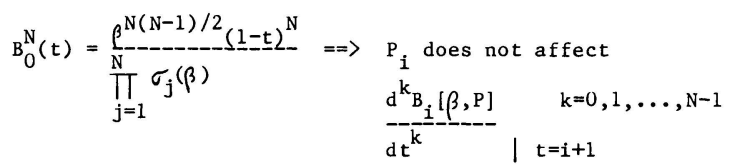

$$
\begin{aligned}
& B_{N}^{N}(t)=\frac{t^{N}}{\prod_{j}^{N} \sigma_{j}(\beta)} \quad \Rightarrow P_{i+N} \text { does not affect } \\
& \frac{d^{k} B_{i}[\beta, P]}{d t^{k}} \mid t=i \\
& \text { 4. Relationship } \Rightarrow \text { Recursion Formula } \quad \Rightarrow \text { Geometric Construction } \\
& \begin{array}{ll}
N \text { and first } & B_{j}^{N+1}(t)=f_{j}^{N}(t) B_{j}^{N}(t) \quad \text { Algorithm }
\end{array} \\
& \mathrm{N}+1 \text { picks } \\
& +s_{j-1}^{N}(t) B_{j-1}^{N}(t) \quad \quad P_{j}^{0}(t)=P{ }_{i+j} \quad 0 \leq j \leq N \\
& P_{j}^{\mathrm{L}}(t)=f_{j}^{N-L}(t-i) P_{j}^{L-1}(t) \\
& +s_{j}^{N-L}(t-i) P_{j+1}^{L-1}(t) \\
& \text { Then } B[\beta, P](t)=P_{0}^{N}(t) \\
& \text { 5. Recursion Formula } \Rightarrow \text { Polynomial Functions } \Rightarrow \text { Polynomial Spline } \\
& =\Rightarrow \begin{array}{l}
\text { Differentiability } \\
\text { Constraints }
\end{array}
\end{aligned}
$$

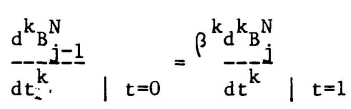

